\title{
La cooperación en ciencia y tecnología entre Argentina y los países de América Latina. El caso del Ministerio de Ciencia, Tecnología e Innovación Productiva (2007-2015)
}

María Paz López*

\begin{abstract}
Resumen
El presente artículo se propone realizar una primera aproximación sistemática a las iniciativas recientes de cooperación científico-tecnológica de la Argentina con países de América Latina, a partir de la creación del Ministerio de Ciencia, Tecnología e Innovación Productiva (MINCYT). Más precisamente, examina los lineamientos de política y los instrumentos a través de los cuales el MINCYT desarrolla la cooperación bilateral con países latinoamericanos durante el período 2007-2015. La discusión de los resultados se da en el marco de la política científico-tecnológica y de la política exterior argentina, caracterizadas por colocar a la cooperación internacional con América Latina como eje prioritario para el país. Las conclusiones advierten diversas iniciativas de colaboración científico-tecnológica de Argentina con países latinoamericanos en temáticas relevantes para los países involucrados.
\end{abstract}

Palabras clave: ciencia - tecnología - cooperación internacional - instrumentos - políticas - Argentina América Latina

\section{Cooperation in science and technology between Argentina and Latin American countries The case of Ministry of Science, Technology and Productive Innovation (2007-2015)}

\begin{abstract}
The present article proposes a first systematic approach to the recent initiatives of scientific and technological cooperation with Latin American countries, since the creation of the Ministry of Science, Technology and Productive Innovation (MINCYT) of Argentina. More precisely, it studies the policy guidelines and instruments through which the MINCYT develops bilateral cooperation with Latin American countries during the period 2007-2015. The discussion of the results comes within the framework of the science and technology policy and the foreign policy in Argentina, characterized by placing international cooperation with Latin America as a priority axis for the country. The conclusions indicate different initiatives of scientific-technological collaboration of Argentina with Latin American countries in topics which are relevant to the countries involved.
\end{abstract}

Key words: science - technology - international cooperation - instruments - policies - Argentina - Latin America

Trabajo enviado: 09/05/2017 Trabajo aceptado: 04/09/2017

\footnotetext{
* Doctora en Ciencias Sociales, Universidad Nacional de La Plata (UNLP, Argentina); Integrante del Centro de Estudios Interdisciplinarios en Problemáticas Internacionales y Locales (CEIPIL-UNICEN-CICPBA); Becaria Posdoctoral del CONICET. Contacto: mpaz_lo@yahoo.com.ar
} 


\section{Introducción}

Desde un punto de vista histórico, Russell y otros (2007) advierten que la cooperación científico-tecnológica en América Latina se ha caracterizado por el establecimiento de lazos de colaboración con los Estados Unidos y Europa, los cuales concentran la mayor cantidad de recursos económicos, humanos y materiales requeridos para el desarrollo de la investigación científico-tecnológica. En datos concretos, Sancho y otros (2006) afirman que entre 1999 y 2002 se incrementó la colaboración internacional y nacional en las publicaciones de todas las áreas científicas de los países de América Latina. Sin embargo, dicho incremento no se dio de igual manera en las co-publicaciones regionales, manteniéndose prácticamente sin variación y a niveles muy bajos en el período estudiado por los autores - pasando de 2,7\% en 1991-1995 a $3 \%$ en 1999-2002.

Los científicos e instituciones latinoamericanas han prestado poca atención a la colaboración con los vecinos más próximos. Esto ha sucedido a pesar de su cercanía en términos históricos, lingüísticos y culturales así como de las problemáticas comunes en temas de salud, agrícolas, ecológicos y geofísicos (Russell y otros, 2007). Además, han existido pocos y diferenciados esfuerzos por establecer un sistema científico-tecnológico en los distintos países de la región. Los investigadores latinoamericanos han contado con pocos recursos intelectuales, materiales y financieros para ofrecer a sus colegas (Velho, 2000). No obstante, es preciso señalar la existencia de experiencias exitosas de cooperación entre países latinoamericanos como es el caso de la colaboración entre Argentina y Brasil iniciada en 1986, a partir de la creación del Centro Argentino-Brasileño de Biotecnología, el cual continúa en funcionamiento (Albornoz; Gordon, 2011).

Ahora bien, los inicios del siglo XXI estuvieron asociados a un cambio de época para los países latinoamericanos, en especial para los de América del Sur. Por entonces, en varios países de la región se produjo la elección de nuevos gobiernos comprometidos con la construcción de proyectos políticos, económicos y sociales alternativos al neoliberalismo, el cual había gozado de plena hegemonía durante los últimos veinte años del siglo XX (Lechini, 2014). Estas circunstancias se dieron con distinta intensidad en Venezuela, Brasil, Bolivia, Paraguay, Ecuador, Uruguay, Chile y Argentina. En Argentina, el proceso estuvo liderado por Néstor Kirchner (2003-2007) y Cristina Fernández de Kirchner (2007-2015) (Colombo, 2011; Lorenzini, 2013). En este marco, Argentina promovió la ampliación de la agenda de integración con países latinoamericanos a partir de la incorporación de nuevas dimensiones y nuevos temas como los de ciencia y tecnología (Araya y otros, 2015).

El presente trabajo se propone realizar una primera aproximación sistemática a las recientes iniciativas de cooperación científico-tecnológica con países de América Latina existentes en el Ministerio de Ciencia, Tecnología e Innovación Productiva (MINCYT) de Argentina, a partir de su creación en el 2007 y hasta el año 2015, en el marco de la política de ciencia y tecnología y exterior del período. En este contexto, señala las principales características de la cooperación internacional con los países latinoamericanos a partir del análisis de documentos oficiales gubernamentales. Específicamente, se pregunta cuáles son y qué características tienen los lineamientos de política y los instrumentos específicos diseñados e implementados para dar curso a la colaboración bilateral con contrapartes latinoamericanas en ciencia y tecnología desde el MINCYT. A partir de allí se interroga cuál es el papel atribuido por el Estado a la cooperación científico-tecnológica con los países de América Latina en relación con otras políticas públicas, como la científico-tecnológica y la exterior. Con este trabajo se espera aportar al estudio de la cooperación científico-tecnológica internacional desde el punto de vista de la política pública y el rol del Estado en su promoción y orientación ${ }^{1}$. En términos generales, pretende contribuir al estudio de políticas públicas recientes.

\footnotetext{
${ }^{1}$ En trabajos anteriores se han caracterizado las dimensiones internacionales de la investigación (LÓPEZ, 2015) y se ha avanzado en una indagación micro-sociológica sobre las relaciones entre prácticas internacionales y políticas científico-tecnológicas (LOPEZ, 2016).
} 


\section{La cooperación internacional en ciencia y tecnología como política pública}

La cooperación internacional constituye una actividad fundamental en el ámbito de la ciencia y la tecnología. La cooperación científico-tecnológica es definida como el conjunto de actividades que, a través de diversos actores y de múltiples instrumentos, "implican una asociación y colaboración para la consecución de objetivos acordados conjuntamente, así como para la obtención de un beneficio mutuo en el ámbito de la investigación, el desarrollo científico-tecnológico y la innovación" (Sebastián; Benavides, 2007:94). Por su parte, la cooperación internacional es entendida como una forma específica de dicha colaboración, realizada entre actores e instituciones de distintos países. En los últimos veinte años se advierte una mayor intensidad y alcance del fenómeno, asociado a los procesos de globalización, el desarrollo de las nuevas tecnologías de la información y la comunicación y la ampliación del espectro de instrumentos destinados a su promoción (Kleiche-Dray; Villavicencio, 2014).

Sebastián (2004) señala la existencia de dos modelos de cooperación internacional en ciencia y tecnología: uno "espontáneo" y otro "integrado". En el primero, la cooperación es llevada adelante por profesores e investigadores de forma espontánea, pasiva e individual y se desarrolla con el objetivo principal de obtener financiamiento. En el segundo caso, la cooperación se presenta como un elemento estratégico integrado a un plan de acción donde se definen los objetivos, políticas e instrumentos propios así como los criterios de selectividad de los socios más adecuados para garantizar el beneficio mutuo. Además, mientras el modelo espontáneo cuenta únicamente con financiación externa, haciendo de la cooperación internacional una actividad imprevisible e impuesta exógenamente, en el modelo integrado las fuentes de financiamiento son mixtas. Esto genera mayor estabilidad así como también una mayor capacidad de negociación.

Desde su perspectiva, Hurtado (2012) advierte que la cooperación internacional puede enmarcarse dentro de una política nacional de desarrollo científico-tecnológico de largo plazo, integrando la actividad científica al desarrollo social y económico. A partir de esta integración y planificación, pueden establecerse objetivos y criterios de diversa índole (políticos, estratégicos, económicos y académicos) para seleccionar las iniciativas de colaboración internacional más convenientes al desarrollo científico-tecnológico nacional. Losego y Arvanitis (2008) advierten que la mayoría de los países carecen de instrumentos financieros capaces de actuar sobre las principales tendencias en la producción de conocimiento en el mundo. Sin embargo, pueden orientar las actividades de investigación hacia temáticas relacionadas con problemáticas locales y privilegiar las cooperaciones que resulten más respetuosas de los intereses definidos localmente.

La política científico-tecnológica promueve capacidades y estipula objetivos a partir de los cuales el país se proyecta internacionalmente en materia científico-tecnológica, atrae socios en el exterior y desarrolla actividades de cooperación internacional. Específicamente, las políticas científico-tecnológicas cumplen dos objetivos: por una parte, promueven la constitución de capacidades de investigación, desarrollo tecnológico e innovación, las cuales resultan fundamentales al momento de cooperar en el ámbito internacional y constituirse en socios atractivos (Kivyk, 2012). Por otra, establecen los objetivos políticos, sociales y económicos más amplios que resultan de interés para un gobierno y que funcionan como guías de las distintas actividades de investigación, desarrollo tecnológico e innovación realizadas en el país, incluidas las de cooperación internacional en el sector científico-tecnológico.

Cabe destacar la existencia de políticas específicas de cooperación internacional que estipulan objetivos concretos e instrumentos particulares para promover este tipo de actividades. En este caso, los gobiernos diseñan, ejecutan y financian programas propios de cooperación de acuerdo con objetivos de interés estratégico para el país con la consiguiente financiación. Además, brindan el marco legal para las relaciones bilaterales a través de los convenios intergubernamentales y dan cobertura a la participación del país en organismos y programas internacionales (Sebastián; Benavides, 2007). 
Por su parte, tal como advierte Kern (2014) la cooperación internacional en ciencia y tecnología no sólo se encuentra influida por la política científico-tecnológica, sino también por la política exterior de un país, la cual indica el catálogo de países o regiones con quienes se promueve la cooperación. La política exterior refiere a un programa de decisiones y acciones llevadas a cabo por la dirigencia de un Estado para modificar o preservar las condiciones del contexto internacional y así promover los intereses y valores de ese Estado y crear las condiciones favorables para la estrategia de desarrollo vigente (Colombo, 2011). De esta manera, la forma de inserción internacional de un país resulta de la proyección externa de su modelo de político-institucional y económico social interno. A su vez, conforma una pieza indispensable para consolidar el mismo (Araya y otros, 2015).

En el marco de la cooperación internacional, se habla de cooperación Norte-Sur y SurSur. Estos conceptos desbordan la categoría geográfica y se asientan en una concepción política y económica: el Norte aglutina naciones industrializadas, desarrolladas y centrales mientras que el Sur conjuga a los países en vías de desarrollo, los cuales, aunque heterogéneos, presentan situaciones de vulnerabilidad y desafíos comunes (Lechini, 2014). La cooperación Norte-Sur remite a las acciones emprendidas por los países industrializados para atender las carencias de los países en desarrollo, es decir, adopta la modalidad de asistencia -principalmente técnicapara el desarrollo. Por su parte, la cooperación Sur-Sur remite a una relación de mayor simetría, de trabajo conjunto y de beneficios mutuos para el logro de una idea de desarrollo compartida (Colacrai y otros, 2009). De la misma manera, el concepto de regionalismo implica una posición ideológica, política y constructora de identidad (Kern, 2014).

Una vez delineado el conjunto de dimensiones analíticas que orientan el trabajo, a continuación se exponen las consideraciones metodológicas del mismo.

\section{Decisiones metodológicas para el abordaje del caso del MINCYT}

El presente artículo estudia el caso del MINCYT de la República Argentina, un organismo público dependiente del Poder Ejecutivo Nacional. Cabe señalar que en 2007, a partir de la gestión de gobierno de Cristina Fernández de Kirchner, se creó el MINCYT, responsabilizándose por las políticas de ciencia, tecnología e innovación poniendo en marcha iniciativas para fortalecer las vinculaciones nacionales e internacionales del sistema. Más precisamente, a través de su Dirección Nacional de Relaciones Internacionales, asumió un rol importante en el ámbito de la cooperación internacional, impulsando la vinculación de los investigadores y empresas argentinas con el mundo. El recorte temporal se extiende desde la creación del MINCYT hasta el año 2015. A partir del año 2016, la Dirección Nacional de Relaciones Internacionales dejó de existir y las relaciones internacionales del Ministerio se encuentran actualmente bajo la órbita de la Dirección Nacional de Cooperación e Integración Institucional.

Distintos trabajos se han ocupado de analizar las instancias multilaterales de cooperación científico-tecnológica entre países latinoamericanos ${ }^{2}$. La presente contribución se centra en la cooperación bilateral entre Argentina y un estado extranjero, la cual se origina, según la propia página web oficial del MINCYT, a partir de la firma de acuerdos internacionales e interinstitucionales. El objetivo principal de estas actividades de cooperación consiste en fomentar la vinculación de la comunidad científica nacional con sus pares en el extranjero, contemplando el interés mutuo en desarrollar investigaciones e intercambiar conocimientos. Asimismo, la colaboración se implementa a través de proyectos conjuntos de investigación, centros binacionales, eventos y becas. El MINCYT presenta actividades de cooperación bilateral con cuarenta y dos (42) países. Esta investigación recupera las correspondientes a los países latinoamericanos presentes en la sección de "Cooperación Bilateral" de la página web del MINCYT, los cuales son siete (7): Brasil, Chile, Colombia, Cuba, México, Perú y Uruguay.

\footnotetext{
${ }^{2}$ Véase por ejemplo: Velho, 2000; Marí y otros, 2001; Corder y otros, 2002; Russell y otros, 2007; Aintablan, Macadar, 2009; Zurbriggen, González Lago, 2010; Larrea, Astur, 2011; y Kern, 2014.
} 
El presente trabajo analiza cualitativamente un conjunto de documentos institucionales escritos (López Noguero, 2002). Para ello, recupera un cuerpo de publicaciones gubernamentales relativas a la cooperación bilateral entre Argentina y países latinoamericanos compuesto por cincuenta y tres (53) comunicados de prensa, treinta y un (31) bases de convocatorias y tres (3) textos de acuerdos y protocolos de creación de centros. Estos documentos se obtuvieron de la sección Cooperación Bilateral correspondiente a la página web oficial del Ministerio tal como se encontraba a fines del año 2015. Por una parte, los documentos se analizaron internamente, procurando describir su contenido en términos de lineamientos, actividades, objetivos y temáticas de cooperación bilateral en ciencia y tecnología entre Argentina y las contrapartes latinoamericanas. Complementariamente, se desarrolló un análisis externo (Fernández Chaves, 2002), buscando comprender los documentos en el contexto de la política científico-tecnológica y exterior argentina.

También se trabajó con los dos Planes Nacionales de Ciencia y Tecnología del período y un cuerpo de discursos de Cancilleres en funciones durante el recorte temporal seleccionado, extraídos de la página oficial del Ministerio de Relaciones Exteriores y Culto de la República Argentina (MRECIC). Los resultados obtenidos se examinaron, complementaron y contrastaron con el apoyo de publicaciones académicas pertinentes. A continuación se expone el análisis realizado sobre los documentos.

\section{Lineamientos de política para la cooperación científico-tecnológica con países latinoamericanos en el MINCYT}

El presente apartado busca comprender los lineamientos de política para la cooperación con países latinoamericanos en materia científico-tecnológica brindados desde el MINCYT. Para ello, tiene en cuenta el marco de la política de ciencia y tecnología más amplia, la cual promueve capacidades de investigación, desarrollo e innovación a la vez que estipula objetivos generales para las actividades científico-tecnológicas en su conjunto. A su vez, examina el papel atribuido a la región latinoamericana en el marco de la política científico-tecnológica general y de la política de cooperación internacional en ciencia y tecnología específicamente.

\subsection{Breve caracterización de la política científico-tecnológica del período}

Desde el inicio de la gestión del Presidente Néstor Kirchner en 2003 y durante las gestiones presidenciales consecutivas de Cristina Fernández (2007-2011 y 2011-2015), se proclamó a la Ciencia y la Tecnología como un área central y una política permanente del Estado. El objetivo declarado para el sector fue el de apuntalar el modelo de desarrollo tendiente al crecimiento económico con mayores niveles de justicia social. En principio, el período estudiado se caracterizó por la planificación a largo plazo a través del Plan Estratégico Nacional de Ciencia, Tecnología e Innovación "Bicentenario" (2006-2010) y el Plan Nacional de Ciencia, Tecnología e Innovación “Argentina Innovadora 2020”, este último lanzado en 2012.

Entre los objetivos de ambos planes se destacan los orientados hacia la cuestión económica y los relacionados con la temática social. Así, los planes indicaron que el sector científico-tecnológico debía generar capacidades para agregar valor a los bienes producidos en el país y para innovar, contribuyendo a la instalación de una economía con un fuerte componente de conocimiento. Además, los planes propusieron que las acciones del sector contribuyesen con el aumento de la calidad de vida de la población y con la consecución de niveles razonables de equidad, armonía social y autonomía nacional.

Por otra parte, como ya se mencionó en la sección metodológica, en 2007 se creó el MINCYT, lo cual implicó la presencia directa de la problemática científica y tecnológica en el proceso de toma de decisiones a nivel país. Además, se pusieron en marcha una serie de iniciativas que buscaron fortalecer los recursos humanos del sistema así como las vinculaciones entre los actores, tanto a nivel nacional como internacional. En cuanto al presupuesto destinado por el Estado a la financiación de actividades científico-tecnológicas, se observó un aumento 
considerable de los recursos del sistema, llegando a significar un $0,64 \%$ del PBI para el año 2014, según los datos más actuales de la Red Iberoamericana e Interamericana de Indicadores de Ciencia y Tecnología (RICYT). Por su parte, hubo una preocupación particular por mejorar la infraestructura científico-tecnológica.

Además, se estipularon prioridades concretas para la investigación científica y el desarrollo tecnológico así como para la formación de investigadores, científicos y tecnólogos a través de Áreas-Problema-Oportunidad y Áreas Temáticas Prioritarias en el caso del Plan "Bicentenario" y a través de las áreas temáticas de alto valor estratégico en el caso del Plan "Argentina Innovadora 2020". Éstas reflejaron, en su conjunto, problemas del desarrollo productivo y social y oportunidades emergentes en la producción de bienes y servicios, en los que la investigación científica y el desarrollo de tecnologías, podían aportar soluciones y/o nuevas perspectivas.

En este marco, y de acuerdo al documento Ciencia y Tecnología argentina en el mundo del MINCYT, la cooperación internacional fue considerada un instrumento fundamental para fortalecer y complementar las capacidades nacionales en ciencia y tecnología y para potenciar los esfuerzos nacionales, con vistas en los objetivos económicos y sociales propuestos para el sector. Específicamente, el Plan Nacional de Ciencia, Tecnología e Innovación "Argentina Innovadora 2020" afirmaba que las acciones de cooperación internacional en ciencia y tecnología debían orientarse hacia la profundización de la inserción y participación de los científicos, tecnólogos y empresarios argentinos en el escenario internacional. Asimismo, proponía que las mismas debían contribuir a la generación, adaptación, difusión y transferencia de conocimiento para agregar valor a los productos y servicios nacionales y mejorar la calidad de vida de la población.

3.2. La cooperación internacional con países de América Latina en el marco de la política científico-tecnológica

El "Plan Argentina Innovadora 2020" propuso fortalecer los procesos de cooperación tanto a nivel regional y subregional, teniendo como horizonte a América Latina y como prioridad inmediata al Mercado Común del Sur (MERCOSUR) y la Unión de Naciones Suramericanas (UNASUR). En su eje referido a la "Articulación internacional”, el Plan aspiró a promover lazos a nivel regional con los países del MERCOSUR con vistas a la creación del Espacio MERCOSUR de Ciencia, Tecnología e Innovación. Además, contempló el diseño y definición de una estrategia latinoamericana de cooperación, potenciando el liderazgo argentino y coordinando las agendas de los países con las de los organismos multilaterales, a fin de complementar esfuerzos y recursos.

En el marco del Ministerio y durante el período estudiado, la cooperación internacional en ciencia y tecnología fue vista como un instrumento para hacer frente a los desafíos de la globalización. Así, se consideró que la producción de conocimientos científico-tecnológicos en colaboración internacional debía apuntar a la inclusión del país en la economía globalizada y a la promoción de la sociedad del conocimiento. En este punto, destaca la dimensión económica de la vinculación, asociada a la generación de empresas de base tecnológica. Ahora bien, se consideró que la cooperación en ciencia y tecnología a nivel internacional debía apuntar, asimismo, al logro de sociedades más justas, al incremento de la calidad de vida de las personas y a la generación de una distribución de la riqueza más equitativa. Según los documentos analizados, el desafío de los países latinoamericanos ha sido colocar a la ciencia y la tecnología como motor de desarrollo; a su vez, dichos materiales presentan a la ciencia, la tecnología y la innovación como parte sustantiva en la articulación latinoamericana con el objetivo de generar una relación más fuerte con los grandes bloques de poder a nivel mundial.

Se advierte, además, que la cooperación regional constituyó una prioridad en la política científico-tecnológica argentina durante los años estudiados. De acuerdo con la entonces Directora Nacional de Relaciones Internacionales, Águeda Menvielle, existió "una voluntad política de la Argentina de jerarquizar las relaciones con América Latina en materia científico- 
tecnológica y poder transferir el ejemplo exitoso de nuestro país a otros países de la región" (MINCYT, 2012b). Por una parte, se consideró importante la integración científico-tecnológica de América Latina para cumplir el objetivo de adquirir una masa crítica de recursos humanos y materiales así como para orientar la producción de conocimientos y la formación de investigadores de una manera acorde a las necesidades y problemáticas regionales. Incluso, se resaltó la necesidad de que los profesionales se formasen en la región y no en los grandes centros del hemisferio norte (MINCYT, 2013b). Por otra parte, se asumió como relevante el llevar adelante acciones de cooperación internacional tendientes a la comparación de políticas, resultados e instrumentos para contribuir a la gestión de la ciencia en los países latinoamericanos (MINCYT, 2013c).

Las actividades de cooperación internacional y regional en el ámbito de la ciencia y la tecnología se relacionan con la relevancia adquirida por la ciencia y la innovación en América Latina (Botella; Suárez, 2012). En Argentina, particularmente, se advierte que el Estado amplió, durante el período estudiado, los temas de agenda y las fuentes de financiamiento de la cooperación científico-tecnológica internacional con las naciones latinoamericanas, poniendo especial énfasis en las relaciones con el MERCOSUR (Larrea; Astur, 2011). Así, se trabajó en el fortalecimiento de las relaciones con socios estratégicos y en la cooperación con los países de menor desarrollo a fin de disminuir las asimetrías existentes y lograr una mejor interlocución de la región con otros actores de la comunidad internacional. Además, Argentina buscó posicionarse como referente en Latinoamérica respecto del desarrollo académico y científico (Artz, 2012).

\section{Instrumentos de cooperación científico-tecnológica con países latinoamericanos en el MINCYT}

En el marco del MINCYT, se encontraron diferentes instrumentos de promoción de la cooperación internacional entre Argentina y los países de América Latina: los centros binacionales, los programas de cooperación binacional en ciencia y tecnología y las visitas oficiales y foros científico-tecnológicos.

\subsection{Centros binacionales creados conjuntamente con países latinoamericanos}

Los centros binacionales constituyen uno de los instrumentos de cooperación internacional con países de América Latina propuestos por el Ministerio. El período se caracteriza por la creación de cuatro centros de investigación conjuntos con países de América Latina como México, Brasil, Chile y Cuba. Dichos centros son: el Centro Argentino-Mexicano en Biotecnología (CAMEB) y el Centro Argentino-Mexicano de Nanociencia y Nanotecnología (CAMEN) con México; el Centro Bilateral en Sistemas de Ingenierías y Matemática Aplicada (CACIMA) con Chile y el Centro Binacional Argentino-Cubano de Biotecnología Aplicada al Desarrollo de Vacunas y Fármacos (CACBVaF) con Cuba.

\section{Cuadro $\mathbf{N}^{\circ}$ 1. Centros binacionales creados por Argentina con contrapartes latinoamericanas} (2007-2015)

\begin{tabular}{|c|c|c|}
\hline Año de Creación & Nombre del Centro & País Socio \\
\hline 2009 & $\begin{array}{c}\text { Centro Binacional Argentino-Cubano de Biotecnología Aplicada } \\
\text { al Desarrollo de Vacunas y Fármacos }\end{array}$ & Cuba \\
\hline 2011 & $\begin{array}{c}\text { Centro Argentino-Mexicano } \\
\text { en Biotecnología }\end{array}$ & México \\
\hline 2011 & Centro Argentino-Mexicano de Nanociencia y Nanotecnología & México \\
\hline 2013 & $\begin{array}{c}\text { Centro Bilateral en Sistema de Ingenierías y Matemática } \\
\text { Aplicada }\end{array}$ & Chile \\
\hline
\end{tabular}

Fuente: Elaboración propia a partir de información extraída de la página oficial del MINCYT 
De acuerdo al análisis realizado sobre los protocolos, acuerdos de creación y comunicados oficiales ${ }^{3}$, los centros binacionales se caracterizan por articular polos existentes de generación y difusión de conocimientos en las áreas contempladas. Entre los objetivos de los centros se incluyen la formación, capacitación y perfeccionamiento de recursos humanos, la generación de conocimientos, productos y procesos y el intercambio y la transferencia de conocimientos científico-tecnológicos. Para ello, las modalidades de cooperación previstas son el desarrollo conjunto y coordinado de proyectos de investigación científico-tecnológica; el intercambio y formación de expertos, científicos y técnicos, en el marco de proyectos conjuntos de cooperación; la organización de conferencias, seminarios, cursos de formación, especialización, perfeccionamiento profesional y adiestramiento; la coordinación de redes y utilización de equipos e instalaciones para el desarrollo conjunto de proyectos específicos; y el intercambio de información científica y tecnológica y de políticas y de gestión en el ámbito de referencia.

En particular, se destaca la orientación del trabajo de los centros binacionales hacia aspectos productivos, haciendo hincapié en la articulación entre el sector público y privado. Así, los centros buscan estimular la creación de empresas binacionales para la generación de productos y procesos en el área de su especialidad; el estudio de cuestiones relativas a patentes y propiedad intelectual e industrial para la comercialización de dichos productos y procesos; la implementación de mecanismos para la protección y distribución de los derechos de propiedad intelectual generados en el marco de proyectos desarrollados por los centros; la creación de grupos de trabajo mixtos con empresas para identificar nichos de mercado, productos y desarrollos tecnológicos que sean requeridos por empresas u organismos gubernamentales de ambos países; la divulgación del conocimiento focalizados en el sector productivo que posibilite la vinculación e interacción entre investigadores y empresarios de los dos países; la formación de recursos humanos del sector empresarial; y la elaboración de estudios y propuestas de mecanismos operacionales para integrar el sector público y privado.

Cada parte cuenta con coordinadores nacionales responsables de las actividades del centro en cuestión, quienes se encargan de examinar las posibilidades de cooperación en temas estratégicos para ambas partes y de elaborar un programa de trabajo, identificando las fuentes y mecanismos de financiamiento disponibles. Estos programas son realizados con la asesoría de un comité compuesto por científicos de cada uno de los países y posteriormente sometidos a consideración de las partes. De esta manera, los centros se proponen abordar temas considerados de importancia estratégica para los países vinculados.

\subsection{Programas de cooperación binacional con países de América Latina}

Además de los centros binacionales descritos anteriormente, otra de las modalidades de cooperación internacional desarrolladas desde el MINCYT son los Programas de cooperación binacional a través de los cuales se financia el desarrollo de proyectos conjuntos de investigación y cooperación y la movilidad de recursos humanos. Los proyectos se presentan en convocatorias abiertas simultáneamente en ambos países y se seleccionan de común acuerdo. Algunos de los programas de cooperación binacional se organizan a partir de temáticas específicas; otros reflejan la cooperación entre organizaciones gubernamentales nacionales y

\footnotetext{
${ }^{3}$ Los documentos utilizados fueron el Acuerdo Marco para la Creación del Centro Binacional Argentino-Cubano de Biotecnología Aplicada al Desarrollo de Vacunas y Fármacos - CACBVAF, firmado el 19 de enero de 2009 en La Habana, República de Cuba; el Protocolo entre el MINCYT de la República Argentina y el CONACYT de los Estados Unidos Mexicanos para la creación de un centro virtual de Biotecnología argentino-mexicano, firmado en Ciudad de México el 30 de mayo de 2011; el Protocolo entre el MINCYT de la República Argentina y el CONACYT de los Estados Unidos Mexicanos para la creación de un centro virtual de Nanociencia y Nanotecnología argentinomexicano, firmado en Ciudad de México el 30 de mayo de 2011; el Comunicado de Prensa denominado Barañao participó en la V Reunión Binacional de Ministros de Chile y Argentina, con fecha 23 de agosto de 2013.
} 
extranjeras dedicadas a la promoción de la ciencia y la tecnología en todas sus áreas y disciplinas.

Entre los primeros se encuentran: 1) Programa Binacional de Terapia Celular (PROBITEC) y 2) Programa Bilateral de Energías Nuevas y Renovables (PBENR) con Brasil; 3) Programa de Cooperación en Uso Sustentable de la Biodiversidad Argentino-Peruano (PROBAPE) y 4) Programa de Cooperación en Energías Renovables Argentino-Peruano (PROERE) con Perú. Así, un total de cuatro (4) programas bilaterales se organizan alrededor de temáticas específicas como terapia celular, energías nuevas y renovables y uso sustentable de la biodiversidad. Dos (2) de los programas son desarrollados conjuntamente con Brasil mientras que los dos (2) restantes son llevados adelante entre Argentina y Perú. Las actividades de cooperación buscan promover el intercambio de experiencias, generar y difundir nuevos conocimientos y formar recursos humanos ${ }^{4}$.

Cuadro 2. Listado de programas de cooperación científico-tecnológica desarrollados por Argentina con contrapartes latinoamericanas sobre temáticas específicas de trabajo (2007-2015)

\begin{tabular}{ccc}
\hline Año de creación & Programa bilateral & Contraparte \\
\hline $\mathbf{2 0 0 8}$ & $\begin{array}{c}\text { Programa Bilateral de Energías Nuevas y Renovables } \\
\text { (PBENR) }\end{array}$ & BRASIL \\
$\mathbf{2 0 0 9}$ & $\begin{array}{c}\text { Programa Binacional de Terapia Celular (PROBITEC) } \\
\mathbf{2 0 1 0}\end{array}$ & $\begin{array}{c}\text { Programa de Cooperación en Uso Sustentable de la } \\
\text { Biodiversidad Argentino-Peruano (PROBAPE) 2010 } \\
\text { Programa de Cooperación en Energías Renovables }\end{array}$ \\
Argentino-Peruano (PROERE) & PERÚ \\
& PERÚ \\
\hline
\end{tabular}

Fuente: Elaboración propia en base a datos de la página web oficial del MINCYT

Entre los segundos se encuentran: 1) Programa de Cooperación con el Consejo Nacional de Desarrollo Científico y Tecnológico (CNPq) de Brasil; 2) Programa de Cooperación Científica y Tecnológica MINCYT-Comisión Nacional de Investigación Científica y Tecnológica (CONICYT) de Chile; 3) Programa de Cooperación científico-tecnológico argentino-colombiano MINCYT-COLCIENCIAS; 4) Cooperación con el Ministerio de Ciencia, Tecnología y Medio Ambiente (CITMA) de la República de Cuba; 5) Programa de Cooperación MINCYT-CONACYT de México; 6) Programa de Cooperación científica y tecnológica con el Consejo Nacional de Ciencia, Tecnología e Innovación Tecnológica (CONCYTEC) de Perú y 7) Programa de Cooperación científico-tecnológico argentino-uruguayo MINCYT-MEC.

Estos siete (7) programas bilaterales se organizan alrededor de instituciones gubernamentales encargadas de promover las actividades científico-tecnológicas. Los mismos son desarrollados con contrapartes brasileñas, chilenas, colombianas, cubanas, mexicanas, peruanas y uruguayas. En este caso, se busca la financiación de proyectos de investigación científica y tecnológica desarrollados entre grupos argentinos y contrapartes latinoamericanas en áreas y temas de interés común y específico. El proyecto de investigación conjunta es definido como un programa acordado entre dos o más grupos de investigación de los países involucrados, con objetivos a alcanzar claramente definidos dentro de un cronograma establecido, e implementado a través de la movilidad de investigadores entre ambos países.

\footnotetext{
${ }^{4}$ Los documentos utilizados fueron el Comunicado de prensa del MINCYT denominado "Barañao firmó acuerdos en biodiversidad y energías renovables en Perú", con fecha 22 de marzo de 2010; el comunicado de prensa del MINCYT denominado "Argentina y Brasil financiarán proyectos conjuntos de investigación en células madre", con fecha 13 de noviembre de 2009; las "Bases de la Convocatoria a Proyectos de Investigación Científica y Tecnológica PICT PROBITEC 2011"; Las Bases de convocatoria "PID PROBITEC 2011"; y la caracterización proporcionada para cada Programa en la página oficial del MINCYT.
} 
Así, el intercambio de investigadores, docentes universitarios y becarios de postgrado a nivel de maestría y doctorado se da en el marco de proyectos conjuntos de investigación científica y tecnológica. Dichos intercambios reciben el nombre de "misiones". Además, se promueve la ejecución de proyectos conjuntos que vinculen a los centros de investigación y desarrollo tecnológico con el sector empresarial de los países involucrados. Los llamados son accesibles a todas las áreas del conocimiento, aunque en algunas convocatorias, se priorizan ciertas temáticas. En términos generales, los proyectos presentados atraviesan una evaluación de calidad y pertinencia tanto a nivel nacional como bilateral ${ }^{5}$.

\section{Cuadro 3. Listado de programas de cooperación científico-tecnológica desarrollados entre organismos gubernamentales de Argentina y contrapartes latinoamericanas}

\begin{tabular}{|c|c|}
\hline Programas bilaterales con organismos gubernamentales & Contraparte \\
\hline $\begin{array}{c}\text { Programa de Cooperación con el Consejo Nacional de Desarrollo Científico y } \\
\text { Tecnológico (CNPq) }\end{array}$ & BRASIL \\
\hline $\begin{array}{c}\text { Programa de Cooperación Científica y Tecnológica MINCYT-Comisión Nacional } \\
\text { de Investigación Científica y Tecnológica (CONICYT) }\end{array}$ & CHILE \\
\hline $\begin{array}{l}\text { Programa de Cooperación científico-tecnológico argentino-colombiano MINCYT- } \\
\text { COLCIENCIAS }\end{array}$ & COLOMBIA \\
\hline $\begin{array}{c}\text { Cooperación con el Ministerio de Ciencia, Tecnología y Medio Ambiente } \\
\text { (CITMA) de la República de Cuba }\end{array}$ & CUBA \\
\hline Programa de Cooperación MINCYT-CONACYT & MÉXICO \\
\hline $\begin{array}{c}\text { Programa de Cooperación científica y tecnológica con el Consejo Nacional de } \\
\text { Ciencia, Tecnología e Innovación Tecnológica (CONCYTEC) }\end{array}$ & PERÚ \\
\hline $\begin{array}{c}\text { Programa de Cooperación científico-tecnológico argentino-uruguayo MINCYT- } \\
\text { MEC }\end{array}$ & URUGUAY \\
\hline
\end{tabular}

Fuente: Elaboración propia a partir de información extraída de la página web oficial del MINCYT

\subsection{Visitas oficiales y reuniones con autoridades latinoamericanas}

Además de crearse centros binacionales y de desarrollar programas de cooperación binacional, en el caso de Brasil, Chile, Perú, Cuba, Uruguay y México se encuentran un total de treinta (30) reuniones oficiales entre las altas cúpulas del sector científico-tecnológico, en las cuales se manifestó la intención de fortalecer los lazos en materia de ciencia, tecnología e innovación (MINCYT, 2013a; MINCYT, 2012c), se firmaron convenios específicos que actuaron como marco de las actividades de cooperación binacional (MINCYT, 2009; MINCYT, 2012d) y se estipularon áreas de interés común para el desarrollo de estas actividades conjuntas (MINCYT, 2012e; MINCYT, 2013a). Durante estas reuniones se destacó la existencia de problemáticas comunes entre los países así como la importancia de la cooperación bilateral para la solución de las mismas. Asimismo, se puso de manifiesto la importancia de reforzar mutuamente las capacidades científico-tecnológicas y de apuntalar el desarrollo de la región.

En dichas reuniones se expresó la intención de fortalecer las relaciones bilaterales en materia científica y tecnológica entre los países latinoamericanos teniendo en cuenta que la producción de conocimientos debe ser acorde a las necesidades y problemáticas de la región y que debe apuntar a mejorar la inserción de los países dentro de la economía globalizada. De esta manera, se estipuló la importancia de trabajar cuestiones que beneficien a los países involucrados, conformando una masa crítica de recursos a partir del trabajo conjunto. En este

\footnotetext{
5 Para la caracterización de los programas se utilizaron los siguientes documentos: Bases de la Convocatoria a Proyectos de Investigación Científica y Tecnológica de Cooperación Internacional PICT - Cooperación Internacional ANPCyT (Argentina) - CNPq (Brasil) de 2008; Bases Programa MINCY-CONICYT, convocatorias 2011, 2012 y 2013; Bases de la Convocatoria del Programa de Cooperación MINCYT-COLCIENCIAS, años 2012, 2013, 2014; Bases del Programa de cooperación científico-tecnológico argentino-mexicano MINCYT-CONACYT, años 2007, 2009, 2011, 2013; Bases del Programa de Cooperación Científico-Tecnológico Argentino-Cubano del MINCYT y CITMA; Programa de Cooperación Científica y Tecnológica MINCYT CONCYTEC. Bases de convocatoria; y Programa de Cooperación científico-tecnológica MINCYT MEC. Bases de Convocatoria.
} 
sentido, se puso énfasis en la dimensión económica y social de la cooperación científicotecnológica, entendiendo que la misma debe contribuir a objetivos como la generación de cadenas de valor agregado, el aumento del volumen de producción, la mejora de la calidad de vida, el incremento de los empleos, la complementariedad y negociación en mejores condiciones en el mercado global, la apertura de mercados en otras regiones del mundo no sólo por parte de grandes compañías, la redistribución más equitativa de la riqueza y la construcción de sociedades más justas (Ver por ejemplo: MINCYT, 2008; MINCYT, 2014).

Una preocupación común entre los gestores del sector científico-tecnológico estuvo constituida por la promoción de clústeres empresariales y tecnológicos, la incorporación de valor agregado en la producción de bienes y servicios, el incremento de la inversión por parte del sector privado en empresas de base tecnológica y capital semilla, la formación de profesionales con una visión adecuada a las necesidades productivas de la región y el aumento del trabajo conjunto entre los grupos de investigación, el Estado y el sector productivo. Otro de los tópicos relevantes fue la divulgación, popularización y valoración de la ciencia y la tecnología (Ver por ejemplo: MINCYT, 2009; MINCYT, 2012d).

A lo largo de los distintos encuentros se acordó la importancia de la cooperación latinoamericana en áreas claves para el desarrollo económico como la Nanotecnología y la Biotecnología, estimulando la articulación entre el sector público, privado, académico y productivo. Además, se estipularon temas como el desarrollo en células madre, la aplicación de la Biotecnología a la salud y la inclusión social a través de las tecnologías. Otras áreas de interés comunes para el intercambio científico-tecnológico fueron el turismo, la acuicultura, las tecnologías de la información y la comunicación y la astronomía. También resultaron fundamentales temas como la ingeniería (hidráulica, civil, informática y computación, etc.) y la física de aceleradores.

Un eje importante de intercambio entre los dirigentes del sector se refirió a las buenas prácticas para el fortalecimiento y articulación de políticas e instrumentos de ciencia, tecnología e innovación. Las reuniones oficiales fueron aprovechadas para comparar políticas, evaluar resultados y reflexionar sobre la mejora de la gestión de las ciencias así como también para generar nuevas instancias de formación de recursos humanos en la temática. Algunos encuentros desarrollados tuvieron como objetivo compartir la experiencia argentina de creación de un Ministerio en el área, así como las iniciativas de financiamiento focalizado y de repatriación de científicos. Además, Argentina ha asesorado a científicos y funcionarios latinoamericanos en la participación de proyectos de investigación financiados por la Unión Europea (Ver por ejemplo: MINCYT, 2012b).

Otros temas abordados han sido el papel de la región en el estímulo a la integración y el fortalecimiento de la democracia; la importancia de la cooperación internacional para el fortalecimiento de los países latinoamericanos; la necesidad de integrar a los ministros nacionales en el debate sobre el reacomodamiento de los países de América ante un nuevo orden mundial; el funcionamiento del MERCOSUR con respecto a las demandas y los mecanismos de implementación de acciones científico-tecnológicas; la importancia de profundizar los contactos y armonizar las legislaciones nacionales vigentes para ahondar el proceso de integración regional; y la necesidad de disminuir las asimetrías entre los países de la región.

\section{La cooperación científico-tecnológica con países latinoamericanos en el marco de la política exterior de Argentina}

El presente apartado comprende los lineamientos e instrumentos de cooperación internacional encontrados en el MINCYT en el marco de la política exterior argentina del período. Como se planteó anteriormente, los inicios del siglo XXI estuvieron asociados a un cambio de época para los países latinoamericanos, al producirse la elección de nuevos gobiernos comprometidos con la construcción de proyectos políticos, económicos y sociales alternativos al neoliberalismo. Además, diferentes cambios a nivel internacional y nacional trajeron consigo una revalorización de la cooperación Sur-Sur, de carácter horizontal y respetuosa de los 
intereses particulares de los cooperantes, lo cual atravesó la cooperación científico-tecnológica internacional (Lechini, 2014).

Específicamente, el enfoque Sur-Sur promovió la asociación estados atravesados por semejantes condiciones de desarrollo -a nivel local-, similares desafíos -a nivel global, experiencias y visiones comunes respecto a la construcción y desarrollo de la nación y la región, y vínculos culturales estrechos (Colacrai y otros, 2009). Todo esto facilitó la adecuación de las acciones de colaboración a necesidades y problemáticas comunes, en condiciones de reciprocidad e igualdad (Lemarchand, 2005).

En Argentina, uno de los rasgos centrales de los gobiernos desarrollados entre 2003 y 2015 ha sido la expansión de la economía a partir del papel regulador, promotor y redistribuidor asumido por el Estado, el impulso a la re-industrialización del país, el crecimiento del mercado interno, la expansión y diversificación de las exportaciones, el desendeudamiento externo, la independencia financiera y la recuperación del control de empresas clave por parte del Estado. A nivel político se profundizó el proceso de democratización a través de la ampliación de derechos sociales, civiles y políticos (Araya y otros, 2015). Las transformaciones sucedidas en el escenario nacional tuvieron su correlato en las estrategias de inserción internacional del país.

La política exterior de los gobiernos de Néstor Kirchner y Cristina Fernández se caracterizaron por dos aspectos clave, como son el corte autonomista y el perfil latinoamericanista, íntimamente relacionados con su definición del proyecto de desarrollo (Lorenzini, 2013). Al respecto, Paredes Rodríguez (2014) indica que la política exterior desarrollada durante la Presidencia de Cristina Fernández ha estado caracterizada por "ajustes en la continuidad" de algunos temas presentes en la agenda de la política exterior vigente durante el período gubernamental previo. Así, la política exterior desarrollada entre 2007 y 2015 se caracterizó por la continuidad y profundización de líneas de acción desplegadas por la gestión inmediatamente anterior.

De acuerdo con las características de la política exterior argentina señaladas anteriormente, la autonomía refiere al fin del alineamiento automático con los Estados Unidos y el rechazo de las recetas ortodoxas de los organismos internacionales de crédito (Lorenzini, 2013). Además, los gobiernos comprendidos entre 2003 y 2015 priorizaron las relaciones con el mundo en desarrollo y tuvieron una mayor disposición hacia los acuerdos Sur-Sur (Malacalza, 2015). Ahora bien, una nota típica del período se encuentra constituida por el perfil latinoamericanista de la política exterior, dándose una aproximación de Argentina a su entono inmediato -el MERCOSUR- y a América Latina en general (Busso, 2014; Miranda, 2015). Durante el período analizado, la identificación con los países del sur se volvió una guía para la política exterior y la cooperación internacional (Kern; Weistaubb, 2010).

En Argentina, la unidad regional se constituyó en prioridad de los gobiernos del período estudiado, buscando consolidar un patrón regional de desarrollo inclusivo, alcanzar una mejor inserción internacional a través del MERCOSUR, aumentar el comercio exterior, diversificar los mercados y multiplicar de la oferta exportable. Además, la integración regional incorporó los objetivos de la lucha contra la pobreza y la inclusión social, propugnó el fortalecimiento de la cooperación y de la solidaridad y buscó la concertación de posiciones afines entre los países de América Latina con el objetivo de tener una voz conjunta en la agenda del desarrollo sostenible propuesta por otros organismos internacionales. El impulso brindado por Argentina a los procesos de integración en América del Sur han fortalecido además las estrategias de cooperación internacional científico-tecnológica (Araya y otros, 2015).

En los discursos de los Cancilleres argentinos en función entre 2003 y 2015 se destacan las siguientes cuestiones: en principio, la cooperación internacional aparece como un instrumento para hacer frente a los desafíos de la globalización. Es decir, en un contexto signado por la globalización, la innovación y el acelerado progreso tecnológico, la cooperación se constituye en "uno de los instrumentos más eficaces para contrarrestar los rasgos negativos de este nuevo mundo en el que se acentúan la incertidumbre, los riesgos y las asimetrías" (MRECIC, 2005). Así, el fortalecimiento de la solidaridad y la concertación de posiciones con 
países con los cuales se comparten intereses, valores y visiones, conforman estrategias para mantener y ampliar los márgenes de autodeterminación del país en el contexto de un mundo globalizado.

En segundo lugar, la integración regional constituye una prioridad en la política exterior argentina. Entre los objetivos y acciones de la política exterior argentina se encuentra la mayor inserción y presencia en América Latina. De acuerdo con el discurso del Canciller Bielsa del año 2007, "el MERCOSUR conforma un proyecto estratégico que trasciende el plano económico-comercial y constituye un espacio sudamericano para maximizar la capacidad de la región de incidir de modo efectivo en el escenario internacional" (MRECIC, 2004). Por su parte, el Canciller Timerman en su discurso del 29 de enero de 2015, afirmaba que los Presidentes Néstor Kirchner y Cristina Fernández apostaron a la unidad regional: "El fortalecimiento de las relaciones de amistad entre nuestros países es una prioridad en la política exterior argentina".

En tercer lugar, el intercambio científico-tecnológico se constituye en un objeto para las relaciones bilaterales y regionales. De acuerdo con el discurso del Canciller Taiana del 7 de agosto de 2007 "la Argentina ha demostrado que en el desarrollo de las relaciones bilaterales puede aportar no sólo flujos de comercio sino también intercambio tecnológico y de conocimiento". En el marco de la integración regional, Argentina impulsa decisiones destinadas a afianzar los vínculos en las áreas política, social, cultural, y, en particular, la científica y tecnológica. Con distintos países de la región, se ha profundizado no sólo la cooperación en materia de comercio y el intercambio económico sino también en lo concerniente a la temática científico-tecnológica.

\section{Reflexiones finales}

El artículo abordó la temática de la cooperación internacional en ciencia y tecnología entre Argentina y los países de América Latina, partiendo de un doble diagnóstico: por una parte, una historia signada por colaboraciones científico-tecnológicas llevadas adelante mayormente con países desarrollados; por otra, los inicios del siglo XXI marcados por un cambio de época para los países latinoamericanos por el cual la cooperación científicotecnológica se transformó en un aspecto relevante del proceso de integración regional. En este marco, se indagaron los instrumentos y políticas de cooperación bilateral de Argentina con los países latinoamericanos en materia de ciencia y tecnología. Más precisamente, se abordaron los lineamientos e instrumentos del Ministerio de Ciencia, Tecnología e Innovación Productiva.

Para desarrollar el estudio, se partió de considerar a la cooperación científicotecnológica internacional como una política pública, relacionada con otras políticas como la científico-tecnológica y la exterior. La política científico-tecnológica determina las capacidades para -y los objetivos de- los intercambios internacionales mientras que la política exterior indica el catálogo de países prioritarios para la vinculación y la importancia de la ciencia y la tecnología para las relaciones exteriores del país. Respecto de la política científico-tecnológica, el período se caracterizó por una jerarquización de la ciencia argentina a partir de la formación de recursos humanos, la ampliación del financiamiento, la creación de instituciones, la diversificación de instrumentos, la puesta en relación de la producción de conocimientos con las necesidades económicas y sociales del modelo de país y la definición de áreas prioritarias. En este marco, la cooperación con América Latina fue una prioridad para el Ministerio en cuestión para la coordinación y comparación de políticas, la complementación de capacidades y recursos, la generación de empresas de base tecnológica y valor agregado y el desarrollo de sociedades más justas.

Entre los instrumentos de cooperación bilateral con países latinoamericanos, se encontró la creación de cuatro (4) centros binacionales, se registró un total de once (11) Programas de Cooperación Binacional con países de América Latina y un total de treinta (30) reuniones y visitas oficiales con contrapartes latinoamericanas. Estos instrumentos se caracterizan por articular polos existentes de producción de conocimientos; la generación y difusión de nuevos 
conocimientos; el intercambio y la formación de recursos humanos; la articulación del sector público-privado y la creación de empresas binacionales. La cooperación internacional se enfoca en la solución de problemáticas comunes, el refuerzo de capacidades, la mejora de la inserción de los países en la economía globalizada, la incorporación de valor agregado y la mejora de la calidad de vida de la población. Por último, se abordó el intercambio de buenas prácticas para la gestión de las ciencias y se compartió la experiencia argentina.

Respecto de la política exterior durante el período estudiado, la misma se caracterizó por un corte autonomista y un perfil latinoamericanista. Así, se priorizaron las relaciones con países en desarrollo y los intercambios Sur-Sur. Específicamente, se dio preferencia a las relaciones con América Latina y el MERCOSUR con el objetivo de hacer frente a desafíos socio-económicos del mundo globalizado así como de conformar una voz conjunta en el escenario internacional. En este marco, la ciencia y la tecnología se conformaron en una estrategia de acercamiento a los países latinoamericanos en el marco de la política exterior argentina.

A lo largo del trabajo se dio cuenta de la existencia de diversos instrumentos para promover los vínculos científico-tecnológicos entre Argentina y los países latinoamericanos entre los años 2007 y 2015, lo cual se condice con el rol otorgado a la región en el marco de la política de ciencia y tecnología más amplia así como de la política exterior del país. En próximos trabajos se profundizará el análisis de estos instrumentos a partir de la realización de entrevistas con actores participantes de los centros y programas binacionales. El objetivo será examinar el alcance de estas propuestas realizadas desde la política pública, las cuales han dotado de importancia a los países de la región como cooperantes, destacando áreas de relevancia conjunta y destinando financiamiento específico.

\section{Bibliografía}

AINTABLÁN, G.; MACADAR, O. (2009). La cooperación internacional en ciencia y tecnología, en Educación Superior y Sociedad, 14 (1), pp. 17-25

ALBORNOZ, M.; GORDON, A. (2011). La política de ciencia y tecnología en Argentina desde la recuperación de la democracia (1983 - 2009), en ALBORNOZ, M. y SEBASTIÁN, J. (Eds.), Trayectorias de las políticas científicas y universitarias de Argentina y España, CSIC, Madrid, pp. 1-46

ARAYA, J., NICOLAO, J., HERRERO, M. S. (2015). Reflexiones sobre la proyección internacional de las universidades subnacionales. Oportunidades y desafíos en el marco de la política exterior argentina durante la última década, en ARAYA, J. M. (Comp.), Aportes para los estudios sobre Internacionalización de la Educación Superior en América del Sur, UNCPBA, Tandil, pp. 78-110

ARZT, E. (2012). El Polo Científico Tecnológico de Buenos Aires, en MINCYT, Hacia un mejor aprovechamiento de la cooperación internacional para el fortalecimiento del Sistema Nacional de Ciencia, Tecnología e Innovación. MINCYT, Buenos Aires, pp. 47-48

BOTELLA, C., SUÁREZ, I. (2012). Innovación para el desarrollo en América Latina. Una aproximación desde la cooperación internacional, en Serie Avances de la Investigación, $\mathrm{N}^{\circ}$ 78, Centro de Estudios para América Latina y la Cooperación Internacional, España

BUSSO, A. (2014). Los vaivenes de la política exterior argentina re-democratizada (1983-2013). Reflexiones sobre el impacto de los condicionantes internos, en Estudios Internacionales, 177, pp. 9-33

COLACRAI, M. y otros (2009). Escenarios y desafíos de la cooperación Sur-Sur a 30 años de la Declaración de Buenos Aires, en Documentos de Trabajo sobre Cooperación Sur-Sur, 1, Centro de Investigación en Cooperación Internacional y Desarrollo (CICI+D), Universidad de San Martín, Buenos Aires

COLOMBO, S. (2011). La inserción internacional de Argentina durante la presidencia de Néstor Kirchner: un cambio de época. UNCPBA, Argentina

CORDER, S.; DA COSTA, M. C.; GOMES, E.; VELHO, P. E. (2002). MERCOSUR: cooperación en ciencia y tecnología, en Nueva Antropología, 18(60), pp. 9-28 
FENÁNDEZ CHAVES, F. (2002). El análisis de contenido como ayuda metodológica para la investigación, en Ciencias sociales, 96, pp. 35-53

HURTADO, D. (2012). La colaboración científica en dos ejes de cooperación clave: Sur-Sur (S-S) y Norte-Sur (N-S), en MINCYT, Hacia un mejor aprovechamiento de la cooperación internacional para el fortalecimiento del Sistema Nacional de Ciencia, Tecnología e Innovación, MINCYT, Buenos Aires, pp. 24-27

KERN, A. (2014). La agenda científica y tecnológica en los regionalismos de América Latina, en Conferencia Internacional Conjunta FLACSO-ISA, 23 al 25 de julio de 2014 1, pp.1-22

KERN, A. ; WEISSTAUB, L. (2010). El debate sobre la cooperación Sur-Sur y su lugar en la política exterior de la Argentina, en Revista Española de Cooperación y Desarrollo, 27

KLEICHE-DRAY, M.; VILLAVICENCIO, D. (2014). Cooperación, colaboración científica y movilidad internacional en América Latina, CLACSO, Buenos Aires

KYVIK, S. (2012). Trabajo en red, colaboración y publicaciones como medios de internacionalización de la investigación, en FERNÁNDEZ LAMARRA, N. y MARQUINA, M., El futuro de la profesión académica. Desafíos para los países emergentes, EDUNTREF, Buenos Aires, pp. 318-328

LARREA, M.; ASTUR, A. (2011). Políticas de internacionalización de la educación superior y cooperación internacional universitaria, en Documento de la Secretaría de Políticas Universitarias, Buenos Aires

LECHINI, G. (2014). La cooperación sur-sur en las políticas exteriores de Argentina y Brasil en el siglo $X X I$, UNR Editora, Rosario, pp. 5-14

LEMARCHAND, G. (2005). Políticas de Cooperación en Ciencia, Tecnología e Innovación en América Latina, en LEMARCHAND, G. (ed.), Memorias del Primer Foro Latinoamericano de Presidentes de Comités Parlamentarios de Ciencia y Tecnología, Buenos Aires, 7 y 8 de marzo de 2005

LÓPEZ, M. P. (2015). Aportes para pensar las dimensiones internacionales de la investigación en América Latina. Revista Iberoamericana de Ciencia, Tecnología y Sociedad, 10(30), pp. 173-198

LÓPEZ, M. P. (2016). Investigadores, prácticas internacionales y políticas científico-tecnológicas: reflexiones a partir de un estudio de caso. Cuadernos Latinoamericanos, 27(49), pp. 1-15

LÓPEZ NOGUERO, F. (2002). El análisis de contenido como método de investigación, en Revista de Educación, 4, pp. 167-179

LORENZINI, M. E. (2013). Las relaciones argentino-chilenas 2008-2011: ¿Realidad o ficción de la “alianza estratégica"?, en Si Somos Americanos, 13(1), pp. 39-64

LOSEGO, P.; ARVANITIS, R. (2008). Science in non-hegemonic countries, en Revue d'anthropologie des connaissances, 2(3), pp. 343-350

MALACALZA, B. (2015). Las fuentes internas de la política de cooperación Sur-Sur al desarrollo de Argentina, en Brazilian Journal of International Relations, 4(2), pp. 198-235

MARÍ, M., ESTÉBANEZ, M. E.; SUÁREZ, D. (2001). La cooperación en ciencia y tecnología de Argentina con los países del MERCOSUR, en Redes, 8(17), pp. 59-82

MIRANDA, R. (2015). El trazo fino de las relaciones bilaterales de Argentina en Sudamérica, en Revista Enfoques, 13(22), pp. 67-81

PAREDES RODRIGUEZ, R. (2014). Medio Oriente en la Política Exterior Argentina: del equilibrismo a los giros en las acciones externas, en BOLOGNA, A. B. (comp.), La política exterior de Cristina Fernández al finalizar su mandato, UNR Editora, Rosario, pp. 351-382

RUSSELL, J. M., AINSWORTH, S., DEL RÍO, J. A., NARVÁEZ-BERTHELEMOT, N.; CORTÉS, H. D. (2007). Colaboración científica entre países de la región latinoamericana, en Revista española de documentación científica, 30(2), pp. 180-198

SEBASTIÁN, J.; BENAVIDES, C. (2007). Ciencia, tecnología y desarrollo. Agencia Española de Cooperación Internacional, España

SEBASTIÁN, J. (2004). Cooperación e internacionalización de las universidades. Biblos, Argentina 
Cuadernos de Política Exterior Argentina (Nueva Época), 126, julio-diciembre 2017, pp. 31-46

ISSN 0326-7806 (edición impresa) - ISSN 1852-7213 (edición en línea)

SANCHO, R.; MORILLO, F.; DE FILIPPO, D.; GÓMEZ, I.; FERNÁNDEZ, M. T. (2006). Indicadores de colaboración científica inter-centros en los países de América Latina, en Revista Interciencia, 31(4), pp. 284-292

VELHO, L. (2000). Redes regionales de cooperación en CyT y el MERCOSUR, en Redes, 7(15), pp. 112-130

ZURBRIGGEN, C.; GONZÁLEZ LAGO, M. (2010). Análisis de las iniciativas MERCOSUR para la promoción de la Ciencia, la Tecnología y la Innovación. CEFIR, Argentina

\section{Documentos oficiales citados}

MINCYT (2006). Plan Estratégico Nacional de Ciencia, Tecnología e Innovación “Bicentenario” (20062010)

MINCYT (2008). Argentina y Chile participaron del Foro Binacional de Ciencia, Tecnología e Innovación Productiva. 1 de septiembre de 2008

MINCYT (2009). Argentina y Chile firman acuerdos de cooperación científica y tecnológica. 6 de agosto de 2009

MINCYT (2011). Argentina y Colombia firmaron un acuerdo en ciencia y tecnología. 19 de agosto de 2011

MINCYT (2012a). Plan Argentina Innovadora 2020. Plan Nacional de Ciencia, Tecnología e Innovación. Lineamientos estratégicos 2012-2015

MINCYT (2012b). Funcionarios argentinos asesoraron a sus pares peruanos en la creación de un futuro Ministerio de Ciencia. 26 de enero de 2012

MINCYT (2012c). Lino Barañao viajará a Brasil para fortalecer las relaciones bilaterales en ciencia y tecnología. 8 de mayo de 2012

MINCYT (2012d). Barañao recorrió Tecnópolis junto al Ministro de Educación y Cultura de Uruguay. 10 de agosto de 2012

MINCYT (2012e). Apoyan cooperación en nanotecnología con México. 24 de agosto de 2012

MINCYT (2013a). Barañao visita Cuba para fortalecer investigaciones conjuntas en biomedicina. 18 de febrero de 2013

MINCYT (2013b). Barañao inauguró la Reunión de Ministros de América Latina y El Caribe. 17 de junio de 2013

MINCYT (2013c). Barañao participó en la V Reunión Binacional de Ministros de Chile y Argentina. 23 de agosto de 2013

MINCYT (2014). El Ministro de Ciencia recibió al embajador de Cuba. 09 de abril de 2014

MINCYT (2017). Ciencia y Tecnología argentina en el mundo. Disponible en: http://www.mincyt.gob.ar/adjuntos/descargas/Politicas_Cooperacion.pdf.

MRECIC (2004). Discurso del Canciller Bielsa, Presentación del libro La Nación Sudamericana, Consejo Argentino para las Relaciones Internacionales, 07 de octubre de 2004

MRECIC (2005). Discurso del Canciller Bielsa Desarrollo y cooperación en el nuevo milenio, en 2nd. International Relations World Conference, Encuentro académico, 16 de agosto de 2005

MRECIC (2007). Discurso del Canciller Taiana, Rol de la Argentina en la región y en la economía global, en Conferencia de la Americas Society, 07 de agosto de 2007

MRECIC (2015). Discurso del Canciller Timerman, en III Cumbre CELAC, Costa Rica, 29 de enero de 2015 